\title{
Autosomal-dominant adult neuronal ceroid lipofuscinosis caused by duplication in DNAJC5 initially missed by Sanger and whole-exome sequencing
}

\author{
Ivana Jedličková ${ }^{1}$ Maxime Cadieux-Dion $\mathbb{1}^{2,3} \cdot$ Anna Přistoupilová ${ }^{1} \cdot$ Viktor Stránecký ${ }^{1}$ Hana Hartmannová ${ }^{1}$. \\ Kateřina Hodaňová ${ }^{1}$ Veronika Barešová ${ }^{1}$ Helena Hůlková ${ }^{1,4}$ • Jakub Sikora (i) ${ }^{1,4}$ • Lenka Nosková ${ }^{1}$ \\ Dita Mušálková ${ }^{1}$ Petr Vyletalal ${ }^{1}$ Jana Sovová ${ }^{1}$ - Patrick Cossette ${ }^{2} \cdot$ Eva Andermann $^{5} \cdot$ Frederick Andermann $^{5}$. \\ Stanislav Kmoch ${ }^{1} \cdot$ The Adult NCL Gene Discovery Consortium
}

Received: 3 May 2019 / Revised: 29 November 2019 / Accepted: 10 December 2019 / Published online: 9 January 2020

(c) The Author(s), under exclusive licence to European Society of Human Genetics 2020

\begin{abstract}
Adult-onset neuronal ceroid lipofuscinoses (ANCL, Kufs disease) are rare hereditary neuropsychiatric disorders characterized by intralysosomal accumulation of ceroid in tissues. The ceroid accumulation primarily affects the brain, leading to neuronal loss and progressive neurodegeneration. Although several causative genes have been identified (DNAJC5, CLN6, CTSF, GRN, CLN1, CLN5, ATP13A2), the genetic underpinnings of ANCL in some families remain unknown. Here we report one family with autosomal dominant (AD) Kufs disease caused by a $30 \mathrm{bp}$ in-frame duplication in DNAJC5, encoding the cysteine-string protein alpha (CSP $\alpha)$. This variant leads to a duplication of the central core motif of the cysteine-string domain of CSP $\alpha$ and affects palmitoylation-dependent CSP $\alpha$ sorting in cultured neuronal cells similarly to two previously described CSP $\alpha$ variants, p.(Leu115Arg) and p.(Leu116del). Interestingly, the duplication was not detected initially by standard Sanger sequencing due to a preferential PCR amplification of the shorter wild-type allele and allelic dropout of the mutated DNAJC5 allele. It was also missed by subsequent whole-exome sequencing (WES). Its identification was facilitated by reanalysis of original WES data and modification of the PCR and Sanger sequencing protocols. Independently occurring variants in the genomic sequence of DNAJC5 encoding the cysteine-string domain of CSP $\alpha$ suggest that this region may be more prone to DNA replication errors and that insertions or duplications within this domain should be considered in unsolved ANCL cases.
\end{abstract}

These authors contributed equally: Ivana Jedličková, Maxime Cadieux-Dion

Supplementary information The online version of this article (https:// doi.org/10.1038/s41431-019-0567-2) contains supplementary material, which is available to authorized users.

$\triangle$ Stanislav Kmoch

skmoch@lf1.cuni.cz

1 Research Unit for Rare Diseases, Department of Pediatrics and Adolescent Medicine, First Faculty of Medicine, Charles University, Prague, Czech Republic

2 Centre Hospitalier de L'Universite de Montréal, Montréal, QC, Canada

3 Center for Pediatric Genomic Medicine, Children's Mercy Hospital, Kansas City, MO, USA

4 Institute of Pathology, First Faculty of Medicine and General University Hospital, Charles University, Prague, Czech Republic

5 Montreal Neurological Hospital \& Institute, McGill University, Montreal, QC, Canada

\section{Introduction}

Adult-onset neuronal ceroid lipofuscinoses (ANCL) constitute a group of rare genetic diseases characterized clinically by the progressive deterioration of mental and motor functions and histopathologically by the intracellular and ultrastructurally distinct accumulation of autofluorescent lipopigment - ceroid — in the brain and other tissues. Age of onset, spectrum of neurological phenotypes, and disease progression can vary even within families. Clinical heterogeneity of ANCLs is in line with diverse inheritance patterns, increasing number of identified causal genes (e.g., DNAJC5 [1], CLN6 [2], CTSF [3], GRN [4], CLN1 [5], CLN5 [6], ATP13A2 [7]), and various types of causative variants and their combinations (NCL Resource-A Gateway for Batten Disease: http://www.ucl.ac.uk/ncl/new nomenclature.shtml).

Diagnosis of ANCLs is challenging from a clinical, histopathologic, as well as diagnostic perspective. Even 
with recent technological advances [8,9], the causative genetic variants(s) in some ANCL families have not been identified. In order to improve genetic diagnosis in these families, we recently established The Adult NCL Gene Discovery Consortium. Within the Consortium we reviewed clinical and histopathological data and classified recruited cases as definite, probable, or possible ANCL or not meeting the diagnostic criteria for ANCL [10]. ANCL cases were then subjected to candidate gene and whole-exome sequencing (WES) [11, 12].

Here we report and characterize a new variant-a 30 base pair in-frame duplication in DNAJC5, that we have identified in one of the investigated ANCL families. The identification of this variant was particularly challenging. It was initially missed by Sanger sequencing of DNAJC5 and WES, to be identified later by reanalysis of original WES data that were shared within the Consortium. Our work thus also provides a cautionary tale about the challenges in identification of even relatively short insertions and duplications by standard genetic methods.

\section{Materials and methods}

\section{Subjects}

The study protocol was approved by the local Institutional Review Boards and signed informed consent was obtained from all subjects.

The Canadian family was ascertained at the Montreal Neurological Institute, McGill University, Canada based on clinical observation of three affected individuals: a mother and two sons. The mother was diagnosed with Kufs disease at the age of 42 and died at the age of 56 (no clinical details are available). The affected sons presented with seizures, memory loss, and disability (wheelchair bound) at the age of 31 and 34. No biopsy material for pathological evaluation was available at the time of investigation to examine for the presence of typical lipopigment in tissues of affected individuals.

\section{DNA sequencing and variant analysis}

Genomic DNA of the two brothers was extracted from whole blood samples by a standard protocol. Coding regions of DNAJC5 (NG_029805.2) were amplified by PCR from genomic DNA of the two brothers and sequenced by direct Sanger sequencing using the version 3.1 Dye Terminator cycle sequencing kit (ThermoFisher Scientific) with electrophoresis on an ABI 3500XL Avant Genetic Analyzer (ThermoFisher Scientific). Data were analyzed using Sequencing Analysis software (ThermoFisher Scientific).

\section{Exome sequencing}

Exome sequencing was performed using genomic DNA from the two affected brothers (Fig. 1). For DNA enrichment, the Sure Select Human All Exon V4 capture kit (Agilent Technologies, Santa Clara, CA) was used according to the manufacturer's protocol. DNA sequencing was performed on the captured barcoded DNA library via an Illumina HiSeq 2000 system as a pair end library with the read length of $100 \mathrm{bp}$. The resulting FASTQ files were aligned to the human reference genome (hg19) via BWAMEM [13]. After genome alignment, conversion of SAM format to BAM and duplicate removal were performed using Picard Tools (1.129). The Genome Analysis Toolkit, GATK (3.2.2) [14-16] was used for local realignment around indels, base recalibration, variant recalibration, and variant calling (HaplotypeCaller). Variant annotation was performed with SnpEff 3.6 [17] and GEMINI 0.18.2 [18].

\section{In silico analysis of the cysteine-string domain}

Hydrophobicity and palmitoylation potential of the wildtype (wt) cysteine-string domain (CSD) and the CSD carrying the DNAJC5 variant were analyzed with a Kyte-Doolittle algorithm and CSS-Palm 2.0, respectively, as described previously [1].

\section{CSPa-expression vectors}

DNAJC5/CSP $\alpha$ cDNA was amplified by RT-PCR from the affected individuals' leukocytes with specific primers. Resulting PCR products were first cloned into PCR2.1 TOPO TA-cloning vector (Invitrogen) and, after sequencing verification, the cDNA region containing the $30 \mathrm{bp}$ duplication was subcloned into a pEGFP-C1/DNAJC5 wt vector using BstXI and BsmBI restriction sites. The pEGFPC1/DNAJC5_wt, pEGFP-C1/DNAJC5_Leu115Arg, and pEGFP-C1/DNAJC5_Leu116del vectors were generated as described previously [1].

\section{Transient expression of EGFP-CSPa}

cDNA constructs were transfected into CAD5 cells derived from Cath. -a-differentiated (CAD) cells (provided by Sukhvir Mahal, The Scripps Research Institute, Jupiter, FL, USA). Four to seven days before transfection, $1 \times 10^{4}$ cells $/ \mathrm{cm}^{2}$ were seeded with Opti-MEM medium (Opti-MEM; Invitrogen) containing 10\% FBS (HyClone, Logan, UT), 90 units penicillin, streptomycin $/ \mathrm{ml}$. Cells were transfected by either 0.8 or $4 \mu \mathrm{g}$ of plasmid constructs with Lipofectamine 2000 (Invitrogen) in serum and antibiotics free Opti-MEM medium according to the 
A

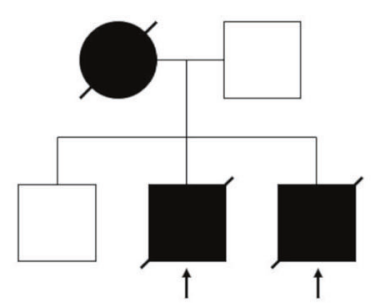

B Proband_sample 1

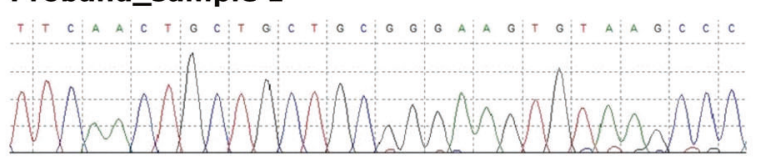

Proband sample 2

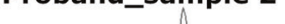

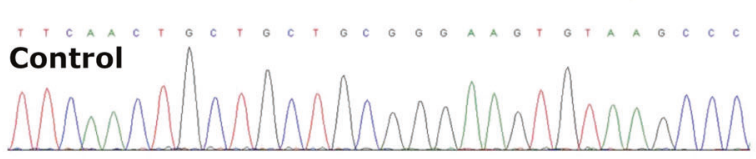

C

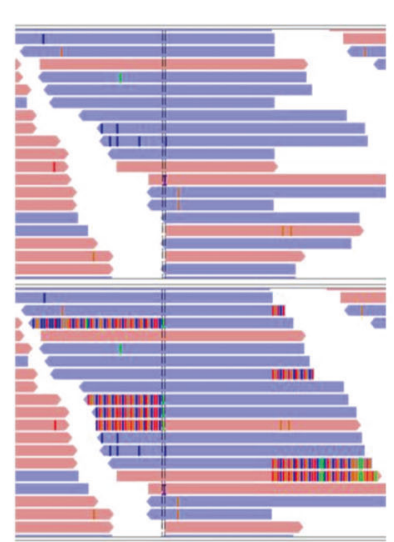

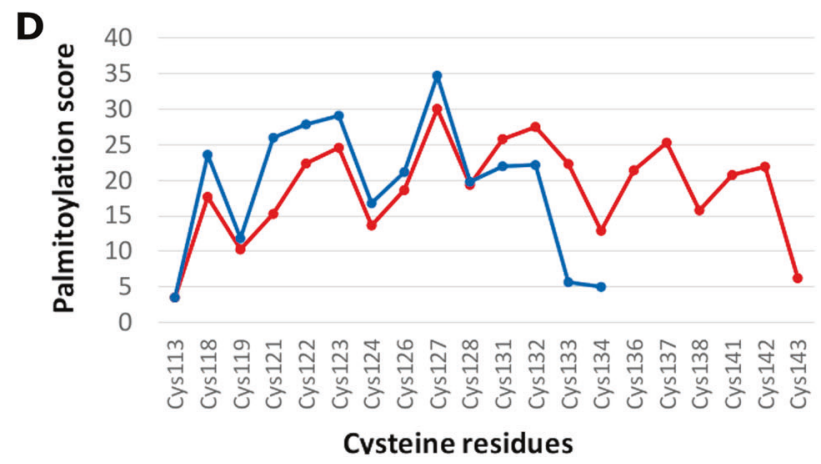

Fig. 1 Sanger sequencing and reads alignment of the DNAJC5 bearing the $30 \mathrm{bp}$ duplication. a Pedigree of the Canadian family suggesting an autosomal dominant inheritance. b Chromatograms of DNAJC5 genomic DNA sequences showing normal DNAJC5 sequence in the proband using original protocol (Proband_sample 1) and heterozygous duplication in the same DNA sample upon modified PCR protocol (Proband_sample 2). Lower panel shows chromatogram from control DNA. c The $30 \mathrm{bp}$ duplication in DNAJC5 in the

manufacturer's protocol. Transfection experiments were performed in more than three replicates.

\section{Immunofluorescence analysis}

Cells were fixed $24 \mathrm{~h}$ after transfection with chilled methanol for $10 \mathrm{~min}$, washed, blocked with $5 \%$ bovine serum albumin (BSA), and incubated for $1 \mathrm{~h}$ at $37^{\circ} \mathrm{C}$ with antiprotein disulfide isomerase mouse monoclonal $\mathrm{IgG} 1$ (Stressgen, San Diego, CA) for endoplasmic reticulum (ER) localization, anti-GS28 mouse IgG1 (Stressgen, San Diego, CA) for Golgi localization, and anti-GFP rabbit polyclonal IgG (Abcam) for EGFP-CSP $\alpha$ detection. For fluorescence detection, corresponding species-specific secondary antibodies Alexa Fluor 488 and Alexa Fluor 555 (Molecular Probes, Invitrogen, Paisley, UK) were used. Prepared slides were mounted in ProLong ${ }^{\circledR}$ Gold Antifade with 4',6-diamidino-2-phenylindole staining nuclei (Life Technologies, Forster City, USA) fluorescence mounting medium and analyzed by confocal microscopy.



\section{Amino acid residues}

Integrative Genomics Viewer (IGV2.3) before (upper panel) and after a visualization of soft-clipped bases (lower panel). $\mathbf{d}$ In silico analysis of the cysteine-string domain showing that compared with the wildtype sequence (blue line), the duplication (red line) alters palmitoylation potential (left panel) and hydrophobicity profile (right panel), critical parameters of post-translational modification, and intracellular localization of CSP $\alpha$.

\section{Image acquisition and analysis}

Prepared slides were analyzed by confocal microscopy. $\mathrm{XYZ}$ images were sampled according to Nyquist criterion using a LeicaSP8X laser scanning confocal microscope, HC PL APO objective (63×, N.A. 1.40), 405, 488, and 543 laser lines. Images were restored using a classic maximum likelihood restoration algorithm in the Huygens Professional Software (SVI, Hilversum, The Netherlands) [19]. The colocalization maps employing single pixel overlap coefficient values ranging from 0 to 1 were created in the Huygens Professional Software [20]. The resulting overlap coefficient values are presented as the pseudo color denoted in the corresponding lookup tables.

\section{Immunoblot analysis}

Transfected CAD5 cells were harvested in PBS, centrifuged at $610 \times g$ for $5 \mathrm{~min}$, resuspended in $50 \mathrm{mM}$ Tris $\mathrm{pH} 6.8$, $50 \mathrm{mM}$ DTT, $2 \%$ sodium dodecyl sulfate (SDS), and Complete Protease Inhibitor Cocktail (Roche) or PBS with 
Triton X-100, 0.1 or $0.5 \%$ and Complete Protease Inhibitor Cocktail (Roche), homogenized by sonication using the Covaris S2 Ultrasonicator, followed by denaturation at $100{ }^{\circ} \mathrm{C}$ for $10 \mathrm{~min}$. The protein content in the supernatant was determined using an infrared spectrometer Direct Detect infrared (Millipore) according to the manufacturer's protocol. Protein lysates equivalent to 15 or $20 \mu \mathrm{g}$ of protein were incubated with and without $6 \mathrm{M}$ hydroxylamine for CSP $\alpha$ depalmitoylation for $24 \mathrm{~h}$ at room temperature and reduced at $100^{\circ} \mathrm{C}$ for $5 \mathrm{~min}$ in sample buffer with or without $1 \%$ beta-mercaptoethanol ( $\beta \mathrm{ME}$ ) before SDSPAGE electrophoresis. After protein transfer to the polyvinylidene fluoride membrane, membranes were blocked by $5 \%$ milk and $0.1 \%$ Tween 20 in PBS over night at $4{ }^{\circ} \mathrm{C}$. CSP $\alpha$ or CSP $\alpha$-EGFP protein was visualized by incubation with rabbit CSP antibody (Stressgen) at 1:5000 in 0.1\% BSA and $0.1 \%$ Tween 20 in PBS for 60 min or rabbit green fluorescent protein (GFP) antibody (Abcam) at 1:3000 in $0.1 \%$ BSA and $0.1 \%$ Tween 20 in PBS for $60 \mathrm{~min}$, followed by incubation with goat antirabbit HRP (Pierce) at 1:10,000 in $0.1 \%$ Tween 20 in PBS for $60 \mathrm{~min}$ and detection by Clarity Western ECL Substrate (Bio-Rad).

\section{Results}

\section{Identification of 30 base pair duplication in DNAJC5 by a combination of exome sequencing and sanger sequencing}

To identify the genetic lesion in affected family members we initially Sanger sequenced and excluded DNAJC5, the prevalent gene for autosomal dominant ANCL (AD-ANCL) (Fig. 1b). Next we sequenced all coding exons and $5^{\prime}$ and $3^{\prime}$ untranslated regions of their corresponding mRNAs (UTRs) (Sure Select Human All Exon V4 capture kit, Illumina HiSeq 2000) in both affected brothers. Considering an autosomal dominant model of inheritance, we searched for variants that had standard read count threshold $\geq 10$, were present in the heterozygous state in both affected individuals and had a minor allele frequency $<0.5 \%$ in The Exome Aggregation Consortium database [21]. These parameters did not yield any functionally relevant candidate variant. Lowering the standard read count threshold to $\geq 5$, we found a $30 \mathrm{bp}$ in-frame duplication in DNAJC5. This variant was however not seen in the IGV tool, which allows visualization of sequence alignments. Essential for the variant detection was a visualization of so called softclipped bases, which are reads not matching with the reference sequence in their whole length. Using the visualization of soft-clipped bases we revealed the $30 \mathrm{bp}$ in-frame duplication: chr20:g.62562252_62562281dup (hg19); NM_025219.2:c.370_399dup (p.(Cys124_Cys133dup)), in exon 4 of DNAJC5. (Fig. 1c). We modified our original PCR protocol and confirmed the presence of the duplication using standard Sanger sequencing (Fig. 1a, upper panels). The variant was submitted to ClinVar database under the accession code VCV000689476 and to the Mutation and Patient Database for Human NCL genes [22].

\section{In silico analysis of the novel CSPa c.370_399dup (p. (Cys124_Cys133dup)) variant}

The duplication encodes for a duplication of the central core motif of the CSD of CSP $\alpha$. NM_025219.2:c.370_399dup (p.(Cys124_Cys133dup)). The CSD is implicated in palmitoylation and membrane trafficking of CSP $\alpha$. In silico analysis suggested that the duplication increases hydrophobicity (Fig. 1d, right panel) of the CSD and that the presence of the additional seven cysteine residues changes the palmitoylation potential (Fig. 1d, left panel). Changes in these parameters can make the protein carrying the $\mathrm{p}$. (Cys124_Cys133dup) variant prone to aggregation [23].

\section{The functional effect of the GFP-tagged CSPa p. (Cys124_Cys133dup) variant in neuronal CAD5 cell model}

To assess the effect of the identified duplication on CSP $\alpha$ expression and intracellular localization we transiently expressed N-terminal GFP tagged CSP $\alpha$ with the identified duplication p.(Cys124_Cys133dup), wt CSP $\alpha$ (GFP_CSP $\alpha$ wt) and GFP_CSP $\alpha$ with previously identified variants NM_025219.2:c.344T $>$ G (p.(Leu115Arg)) and NM_025219.2:c.343_345del (p.(Leu116del)), both variants located in exon 4 of DNAJC5 (the exon numbering starts with exon 1 to exon 5), shortly p.(Leu115Arg) and p. (Leu116del), in CAD5 cells. Immunofluorescence analysis and colocalization studies showed that the GFP_CSP $\alpha$ _wt and the endogenous CSP $\alpha$ are localized dominantly along the plasma membrane in finely granular cytoplasmic structures. All three GFP_CSP $\alpha$ proteins with the variants p. (Cys124_Cys133dup), p.(Leu115Arg), and p.(Leu116del) had reduced expression on the plasma membrane. They are present mostly in cytoplasm, either in a diffuse form or as a coarsely granular inclusions that colocalize to a certain extent with markers of ER and Golgi apparatus (Fig. 2, Supplementary Fig. 1).

\section{Immunoblot analysis of GFP-CSPa transiently produced in CAD5 cells}

To assess the effect of the identified duplication we performed western blot analysis of transiently transfected CAD5 cell lysates before and after chemical depalmitoylation performed under different denaturing conditions 


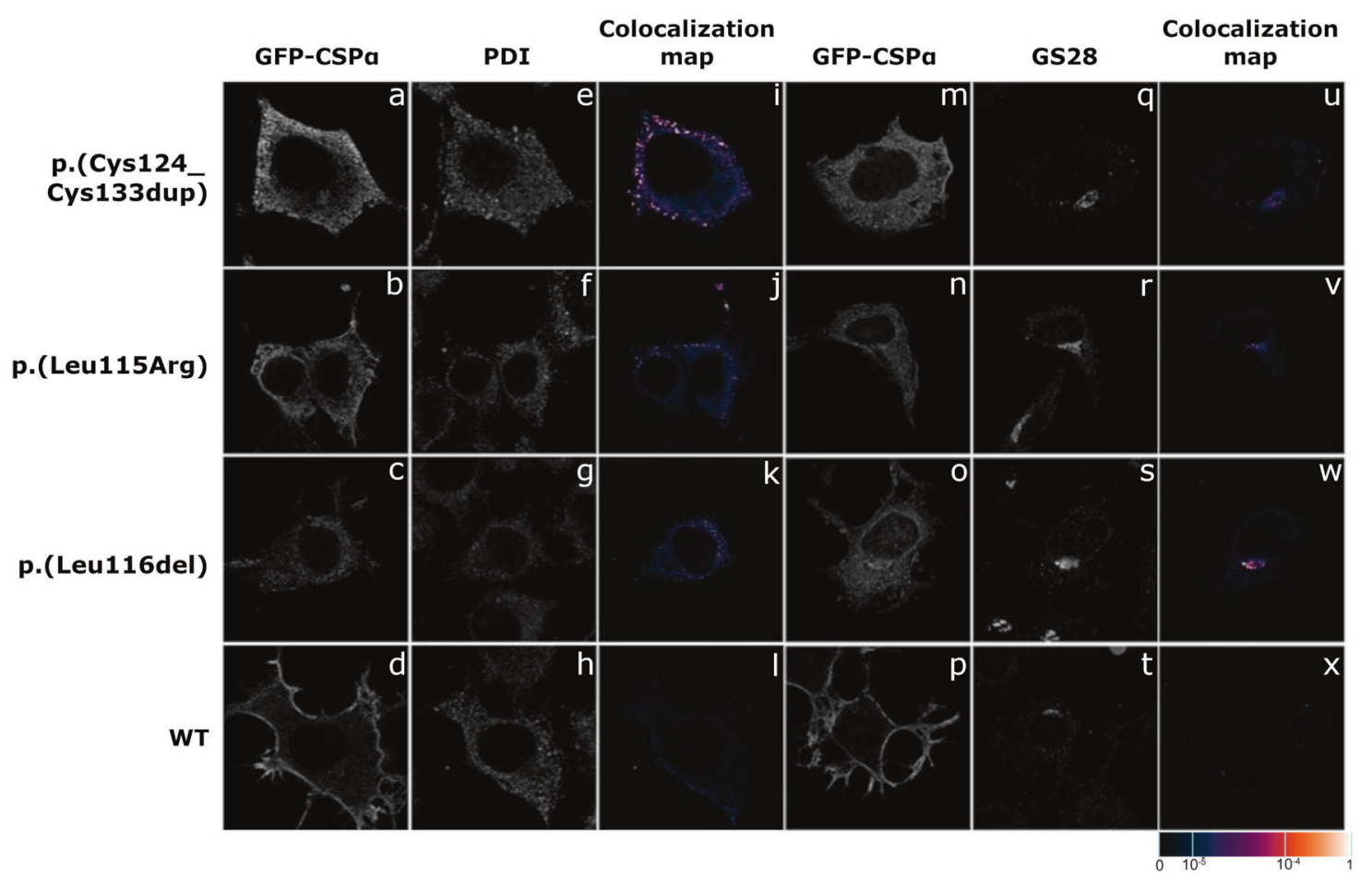

Fig. 2 Immunofluorescence analysis of transiently expressed GFPtagged CSP $\alpha$ wt and variant proteins in CAD5 cells. All three variant proteins $(\mathbf{a}-\mathbf{c}, \mathbf{m}-\mathbf{0})$ are present in a finely or coarsely granular structures. Co-staining with (e-g) protein disulfide isomerase (PDI), a marker of endoplasmic reticulum (ER), and (q-s) Golgi SNAP receptor complex member 1 (GS28) demonstrates abnormal presence

(Fig. 3). We found that the GFP_CSP $\alpha$ protein carrying the Cys124_Cys133dup is present exclusively in nonpalmitoylated form, whereas GFP_CSP $\alpha \_L e u 115 A r g$ and GFP_CSP $\alpha \_L e u 116$ del proteins can present in both, the nonpalmitoylated or palmitoylated CSP $\alpha$ with the former more abundant. All three GFP_CSP $\alpha \_L e u 115$ Arg, GFP_ CSP $\alpha \_L e u 116 d e l$, and GFP_CSP $\alpha \_C y s 124$ Cys133dup proteins formed high molecular weight aggregates that were resistant to SDS and reducing agents (DTT, $\beta \mathrm{ME}$ ). The aggregates became soluble by these procedures only upon initial chemical depalmitoylation by hydroxylamine (Fig. 3).

\section{Discussion}

In this work we identified a 30 bp duplication in DNAJC5 encoding CSP $\alpha$ in one family ascertained by The Adult NCL Gene Discovery Consortium. The variant leads to a duplication of the central core motif of the CSD and affects palmitoylation-dependent CSP $\alpha$ sorting in cultured neuronal cells similar to two other previously described single nucleotide CSP $\alpha$ variants p.Leu115Arg and p.Leu116del. CSP $\alpha$ acts as a co-chaperone in the formation of presynaptic SNARE complexes (soluble N-ethylmaleimide-sensitive of mutated proteins in ER (i-k) and Golgi $(\mathbf{u}-\mathbf{w})$. Wild-type protein $(\mathbf{d}, \mathbf{h}, \mathbf{l}, \mathbf{p}, \mathbf{t}, \mathbf{x})$ is present exclusively on plasma membrane. The degree of colocalization of GFP_CSP $\alpha$ with selected markers is demonstrated by the fluorescent signal overlap coefficient values ranging from 0 to 1 . The resulting overlap coefficient values are presented as the pseudo color whose scale is shown in corresponding lookup table.

factor attachment protein receptors) [24]. The SNAREs are essential for docking of synaptic vesicles, their fusion and recycling. There is accumulating evidence that disruption of the SNARE machinery leads to neurodegeneration [25].

This family remained genetically undefined for decades. Initially, the variant could not be detected by standard Sanger sequencing of DNAJC5 probably due to a preferential PCR amplification of the shorter wt allele and allelic dropout of the mutated DNAJC5 allele. It was also missed by a subsequent analysis of WES. Its identification was facilitated by reanalysis of the original WES data shared within the Consortium and modification of the PCR and Sanger sequencing protocols.

Independently occurring variants in the genomic sequence of DNAJC5 encoding the CSD of CSP $\alpha$ [1] suggest that this region may be more prone to DNA replication errors and that insertions or duplications within this domain should be considered in not yet solved ANCL cases.

Our work demonstrates the limitations of Sanger sequencing and WES in detection of even relatively small insertions and duplications and shows that analysis of next generation sequence data still requires an individualized approach and unique interpretations of the data. Continued reanalysis of the data with a team of experienced scientists 


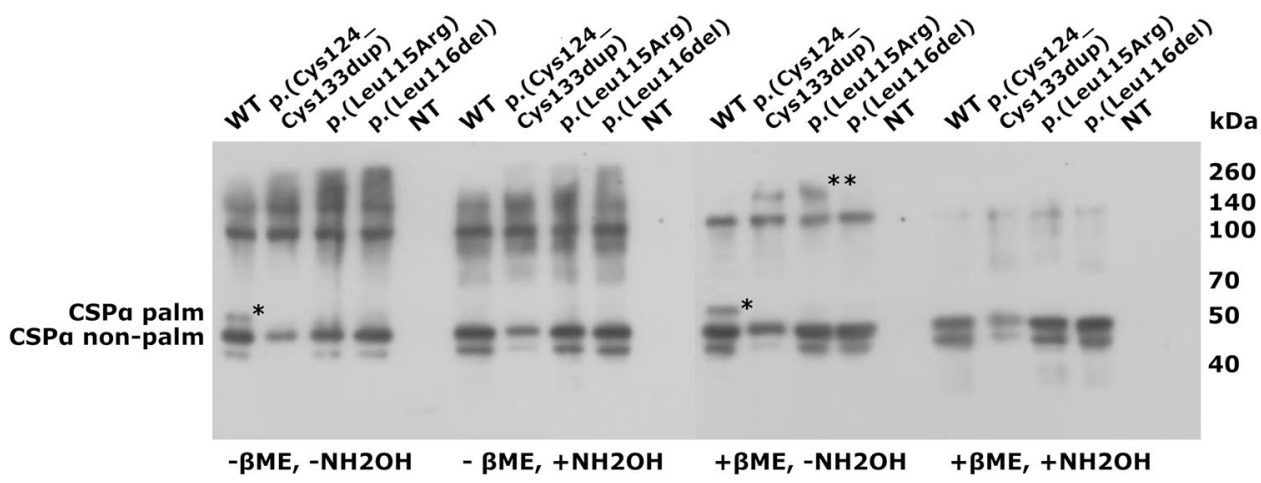

Fig. 3 Immunoblot analysis of transiently expressed GFP-tagged CSP $\alpha$ proteins in CAD5 cells. Immunodetection using the CSP antibody reveals that the GFP_CSP $\alpha \_$p.(Cys124_Cys133dup) protein is present exclusively in nonpalmitoylated form (CSP $\alpha$ nonpalm; the asterisk (*) refers to the palmitoylated form-CSP $\alpha$ palm). All three GFP_CSP $\alpha$ proteins with the variants p.(Leu115Arg), p.(Leu116del), p. (Cys124_Cys133dup) form high molecular weight aggregates $(* *)$ resistant to sodium dodecyl sulfate (SDS) and reducing agents (DTT, $\beta$ ME). The aggregates are soluble only upon initial chemical depalmitoylation by hydroxylamine $(\mathrm{NH} 2 \mathrm{OH})$.

may identify previously missed variants. Approximately $75 \%$ of patients with neurodegeneration subjected to WES remain without a genetic diagnosis [26]. It is unclear how many similar variants will be identified by continued reanalysis, as demonstrated in this paper.

\section{Data availability}

The authors state that anonymized data will be shared by request from any qualified investigator.

Acknowledgements This work was supported by Ministry of Health of the Czech Republic, grant nr. NV19-08-137. Institutional support was provided by Charles University institutional programs PRVOUKP24/LF1/3, UNCE 204064, and SVV2016/260148, by the project LQ1604 NPU II from the Ministry of Education, Youth and Sports of the Czech Republic. We thank The National Center for Medical Genomics (LM2015091) for their instrumental and technical support with the WES analyses. Instrumental support was provided by grant CZ.1.05/2.1.00/19.0400 from the Research and Development for Innovations Operational Programme (RDIOP) cofinanced by European regional development fund and the state budget of the Czech Republic.

\section{Compliance with ethical standards}

Conflict of interest The authors declare that they have no conflict of interest.

Publisher's note Springer Nature remains neutral with regard to jurisdictional claims in published maps and institutional affiliations.

\section{References}

1. Noskova L, Stranecky V, Hartmannova H, Pristoupilova A, Baresova V, Ivanek R, et al. Mutations in DNAJC5, encoding cysteine-string protein alpha, cause autosomal-dominant adultonset neuronal ceroid lipofuscinosis. Am $\mathbf{J}$ Hum Genet. 2011;89:241-52.
2. Arsov T, Smith KR, Damiano J, Franceschetti S, Canafoglia L, Bromhead CJ, et al. Kufs disease, the major adult form of neuronal ceroid lipofuscinosis, caused by mutations in CLN6. Am J Hum Genet. 2011;88:566-73.

3. Smith KR, Dahl HH, Canafoglia L, Andermann E, Damiano J, Morbin M, et al. Cathepsin F mutations cause Type B Kufs disease, an adult-onset neuronal ceroid lipofuscinosis. Hum Mol Genet. 2013;22:1417-23.

4. Smith KR, Damiano J, Franceschetti S, Carpenter S, Canafoglia L, Morbin M, et al. Strikingly different clinicopathological phenotypes determined by progranulin-mutation dosage. Am J Hum Genet. 2012;90:1102-7.

5. van Diggelen OP, Thobois S, Tilikete C, Zabot MT, Keulemans JL, van Bunderen PA, et al. Adult neuronal ceroid lipofuscinosis with palmitoyl-protein thioesterase deficiency: first adult-onset patients of a childhood disease. Ann Neurol. 2001;50:269-72.

6. Xin W, Mullen TE, Kiely R, Min J, Feng X, Cao Y, et al. CLN5 mutations are frequent in juvenile and late-onset non-Finnish patients with NCL. Neurology. 2010;74:565-71.

7. Bras J, Verloes A, Schneider SA, Mole SE, Guerreiro RJ. Mutation of the parkinsonism gene ATP13A2 causes neuronal ceroidlipofuscinosis. Hum Mol Genet. 2012;21:2646-50.

8. Cotman SL, Karaa A, Staropoli JF, Sims KB. Neuronal ceroid lipofuscinosis: impact of recent genetic advances and expansion of the clinicopathologic spectrum. Curr Neurol Neurosci Rep. 2013;13:366.

9. Kmoch S, Stranecky V, Emes RD, Mitchison HM. Bioinformatic perspectives in the neuronal ceroid lipofuscinoses. Biochim Biophys Acta. 2013;1832:1831-41.

10. Berkovic SF, Staropoli JF, Carpenter S, Oliver KL, Kmoch S, Anderson GW, et al. Diagnosis and misdiagnosis of adult neuronal ceroid lipofuscinosis (Kufs disease). Neurology. 2016;87:579-84

11. Ehling R, Noskova L, Stranecky V, Hartmannova H, Pristoupilova A, Hodanova $\mathrm{K}$, et al. Cerebellar dysfunction in a family harboring the PSEN1 mutation co-segregating with a cathepsin D variant p.A58V. J Neurol Sci. 2013;326:75-82.

12. van den Ameele J, Jedlickova I, Pristoupilova A, Sieben A, Van Mossevelde S, Ceuterick-de Groote C, et al. Teenage-onset progressive myoclonic epilepsy due to a familial C9orf72 repeat expansion. Neurology. 2018;90:e658-e663.

13. Li H. Aligning sequence reads, clone sequences and assembly contigs with BWA-MEM. Bioinformatics. 2013;00:1-2. 
14. McKenna A, Hanna M, Banks E, Sivachenko A, Cibulskis K, Kernytsky A, et al. The Genome Analysis Toolkit: a MapReduce framework for analyzing next-generation DNA sequencing data. Genome Res. 2010;20:1297-303.

15. DePristo MA, Banks E, Poplin R, Garimella KV, Maguire JR, Hartl C, et al. A framework for variation discovery and genotyping using next-generation DNA sequencing data. Nat Genet. 2011;43:491-8.

16. Van der Auwera GA, Carneiro MO, Hartl C, Poplin R, Del Angel G, Levy-Moonshine A, et al. From FastQ data to high confidence variant calls: the Genome Analysis Toolkit best practices pipeline. Curr Protoc Bioinform. 2013;43:11 10 1-33.

17. Cingolani P, Platts A, Wang le L, Coon M, Nguyen T, Wang L, et al. A program for annotating and predicting the effects of single nucleotide polymorphisms, SnpEff: SNPs in the genome of Drosophila melanogaster strain w1118; iso-2; iso-3. Fly. 2012;6:80-92.

18. Paila U, Chapman BA, Kirchner R, Quinlan AR. GEMINI: integrative exploration of genetic variation and genome annotations. PLoS Comput Biol. 2013;9:e1003153.

19. Landmann L. Deconvolution improves colocalization analysis of multiple fluorochromes in 3D confocal data sets more than filtering techniques. J Microsc. 2002;208(Pt 2):134-47.
20. Manders EMM, Verbeek FJ, Aten JA. Measurement of colocalization of objects in dual-color confocal images. J Microsc. 1993;169:375-82.

21. Lek M, Karczewski KJ, Minikel EV, Samocha KE, Banks E, Fennell T, et al. Analysis of protein-coding genetic variation in 60,706 humans. Nature. 2016;536:285-91.

22. Mole SE, Gardener E. Mutation and patient database for human NCL genes. 2019. https://www.ucl.ac.uk/ncl-disease/mutation-a nd-patient-database/mutation-and-patient-datasheets-human-nclgenes. Accessed 22 Oct 2019.

23. Diez-Ardanuy C, Greaves J, Munro KR, Tomkinson NC, Chamberlain LH. A cluster of palmitoylated cysteines are essential for aggregation of cysteine-string protein mutants that cause neuronal ceroid lipofuscinosis. Sci Rep. 2017;7:10.

24. Burgoyne RD, Morgan A. Cysteine string protein (CSP) and its role in preventing neurodegeneration. Semin Cell Dev Biol. 2015;40:153-9.

25. Gorenberg EL, Chandra SS. The role of co-chaperones in synaptic proteostasis and neurodegenerative disease. Front Neurosci. 2017;11:248.

26. Fogel BL. Genetic and genomic testing for neurologic disease in clinical practice. Handb Clin Neurol. 2018;147:11-22. 